\title{
Combination of Tocilizumab and Steroids to Improve Mortality in Patients with Severe COVID-19 Infection: A Spanish, Multicenter, Cohort Study
}

\author{
Belén Ruiz-Antorán (D) · Aránzazu Sancho-López · Ferrán Torres • Víctor Moreno-Torres • \\ Itziar de Pablo-López • Paulina García-López • Francisco Abad-Santos • Clara M. Rosso-Fernández • \\ Ana Aldea-Perona · Eva Montané · Ruth M. Aparicio-Hernández · Roser Llop-Rius · Consuelo Pedrós • \\ Paloma Gijón · Carolina Hernández-Carballo · María J. Pedrosa-Martínez · Consuelo Rodríguez-Jiménez • \\ Guillermo Prada-Ramallal · Lourdes Cabrera-García · Josefa A. Aguilar-García • Rocío Sanjuan-Jimenez • \\ Evelyn I. Ortiz-Barraza • Enrique Sánchez-Chica · Ana Fernández-Cruz on behalf of the TOCICOV-study group
}

Received: October 7, 2020 / Accepted: November 16, 2020 / Published online: December 6, 2020

(C) The Author(s) 2020

\section{ABSTRACT}

Background: We aimed to determine the impact of tocilizumab use on severe COVID-19 (coronavirus disease 19) pneumonia mortality.

Belén Ruiz-Antorán and Aránzazu Sancho-López contributed equally to this work.

Electronic supplementary material The online version of this article (https://doi.org/10.1007/s40121020-00373-8) contains supplementary material, which is available to authorized users.

B. Ruiz-Antorán $(\bowtie) \cdot$ A. Sancho-López Clinical Pharmacology Department, Hospital Universitario Puerta de Hierro-Majadahonda, Instituto de Investigación Sanitaria Puerta de Hierro-Segovia de Arana, Madrid, Spain e-mail: bruizantoran@gmail.com

F. Torres

Medical Statistics Core Facility, August Pi i Sunyer Biomedical Research Institute, Hospital Clinic, Barcelona, Spain

F. Torres

Biostatistics Unit, Faculty of Medicine, Autonomous University of Barcelona, Barcelona, Spain

V. Moreno-Torres · E. Sánchez-Chica Internal Medicine Department, Hospital Universitario Puerta de Hierro-Majadahonda, Instituto de Investigación Sanitaria Puerta de Hierro-Segovia de Arana, Madrid, Spain
Methods: We performed a multicentre retrospective cohort study in 18 tertiary hospitals in Spain from March to April 2020. Consecutive patients admitted with severe COVID-19 treated with tocilizumab were compared to patients not treated with tocilizumab, adjusting by inverse probability of the treatment weights (IPTW). Tocilizumab's effect in patients receiving steroids during the $48 \mathrm{~h}$ following inclusion was analysed.

Results: During the study period, 506 patients with severe COVID-19 fulfilled the inclusion

I. de Pablo-López

Clinical Pharmacology Unit, Hospital Universitario

Ramón y Cajal (IRYCIS), Madrid, Spain

P. García-López

Pneumology Department, Hospital Universitario

Torrecardenas, Almería, Spain

F. Abad-Santos

Clinical Pharmacology Department, Hospital Universitario de la Princesa, Instituto Teófilo Hernando, Faculty of Medicine, Universidad Autónoma de Madrid (UAM), Instituto de Investigación Sanitaria la Princesa (IP), Madrid, Spain

C. M. Rosso-Fernández

Unit of Clinical Pharmacology, Unit of Clinical

Investigation and Clinical Trials, Hospital

Universitario Virgen del Rocío, Sevilla, Spain 
criteria. Among them, 268 were treated with tocilizumab and 238 patients were not. Median time to tocilizumab treatment from onset of symptoms was 11 days [interquartile range (IQR) 8-14]. Global mortality was $23.7 \%$. Mortality was lower in patients treated with tocilizumab than in controls: $16.8 \%$ versus $31.5 \%$, hazard ratio (HR) 0.514 [95\% confidence interval (95\% CI) 0.355-0.744], $p<0.001$; weighted HR 0.741 (95\% CI $0.619-0.887), p=0.001$. Tocilizumab treatment reduced mortality by $14.7 \%$ relative to no tocilizumab treatment [relative risk reduction (RRR) 46.7\%]. We calculated a number necessary to treat of 7 . Among patients treated with steroids, mortality was lower in those treated with tocilizumab than in those treated with steroids alone $[10.9 \%$ versus $40.2 \%$, HR $0.511 \quad$ (95\% CI $0.352-0.741)$, $p=0.036 ; \quad$ weighted HR $0.6 \quad(95 \% \quad$ CI

\section{A. Aldea-Perona}

Clinical Pharmacology Department, Consorcio Parc

Salut MarInstituto Hospital del Mar de

Investigaciones Médicas (IMIM)Universidad

Autónoma de Barcelona (UAB), Barcelona, Spain

E. Montané

Clinical Pharmacology Department, Hospital

Universitario Germans Trias i Pujol, Barcelona, Spain

E. Montané

Department of Pharmacology, Therapeutics and

Toxicology, Barcelona, Spain

R. M. Aparicio-Hernández

Clinical Pharmacology Department, Hospital

Universitario Central de La Defensa Gómez Ulla,

Madrid, Spain

R. Llop-Rius

Clinical Pharmacology Department, Hospital

Universitari de Bellvitge, Barcelona, Spain

C. Pedrós

Clinical Pharmacology Unit, Consorci Hospital

General Universitari de València, Valencia, Spain

P. Gijón

Clinical Microbiology and Infectious Diseases

Department, Hospital General Universitario

Gregorio Marañón, Instituto de Investigación

Sanitaria Gregorio Marañón, Madrid, Spain
0.449-0.804), $\quad p<0.001] \quad$ (interaction $p=0.094)$.

Conclusions: These results show that survival of patients with severe COVID-19 is higher in those treated with tocilizumab than in those not treated and that tocilizumab's effect adds to that of steroids administered to non-intubated patients with COVID-19 during the first $48 \mathrm{~h}$ of presenting with respiratory failure despite oxygen therapy. Randomised controlled studies are needed to confirm these results.

Trial registration: European Union electronic Register of Post-Authorization Studies (EU PAS Register) identifier, EUPAS34415

Keywords: COVID-19; Mortality; SARS-CoV-2; Steroids; Tocilizumab

C. Hernández-Carballo Internal Medicine Department, Hospital Universitario Nuestra Señora Candelaria, Tenerife, Spain

M. J. Pedrosa-Martínez

Clinical Pharmacology Department, Hospital

Universitario Puerto Real, Cádiz, Spain

C. Rodríguez-Jiménez

Clinical Pharmacology Department, Hospital

Universitario de Canarias, Tenerife, Spain

C. Rodríguez-Jiménez

Departamento de Medicina Física y Farmacología, Facultad de Medicina, Universidad de La Laguna

(ULL), Tenerife, Spain

G. Prada-Ramallal Epidemiology, Statistics and Research Methodology Unit, Institute for Health Research Foundation (FIDIS), Santiago de Compostela, Spain

L. Cabrera-García

Clinical Pharmacology Department, Hospital

Universitario Clínico San Carlos, Madrid, Spain

J. A. Aguilar-García

Internal Medicine Department, Hospital Costa del

Sol Marbella, Málaga, Spain

R. Sanjuan-Jimenez

UICEC IBIMA, Plataforma SCReN, Málaga, Spain 


\section{Key Summary Points}

Over-exuberant cytokine release occurred in some patients infected by SARS-CoV-2. Treatment strategies aimed to break down this hyper-inflammatory response were considered.

This study describes the experience with a series of consecutive COVID-19 patients treated with TCZ in 18 tertiary hospitals in Spain and compares the outcomes of this cohort with those observed in a similar cohort of SARS-CoV-2 infected patients who did not receive tocilizumab.

Tocilizumab treatment reduced mortality by $14.7 \%$ relative to no tocilizumab treatment (RRR 46. 7\%). Tocilizumab with steroid- $48 \mathrm{~h}$ treatment reduced mortality by $29.1 \%$ relative to no tocilizumab treatment (RRR 72.8\%).

Mortality is improved when tocilizumab is used concomitantly or shortly followed by steroids (first $48 \mathrm{~h}$ ). These results contribute to the body of evidence supporting the use of tocilizumab in SARSCoV-2 infection.

Randomised controlled studies are needed to confirm these results and establish the potential place of tocilizumab in the treatment of COVID-19.

R. Sanjuan-Jimenez

Servicio de Farmacología Clínica, Hospital

Universitario Virgen de la Victoria, Málaga, Spain

E. I. Ortiz-Barraza

Internal Medicine Department, Hospital

Universitario Ramón y Cajal (IRYCIS), Madrid, Spain

\section{A. Fernández-Cruz ( $₫)$}

Infectious Diseases Unit, Internal Medicine

Department, Hospital Universitario Puerta de

Hierro-Majadahonda, Instituto de Investigación

Sanitaria Puerta de Hierro-Segovia de Arana, Madrid, Spain

e-mail: anafcruz999@gmail.com

\section{DIGITAL FEATURES}

This article is published with digital features, including a summary slide, to facilitate understanding of the article. To view digital features for this article go to https://doi.org/10.6084/ m9.figshare.13228859.

\section{INTRODUCTION}

In the past few decades, newly evolved coronaviruses have posed a global threat to public health.

The most recent one, SARS-CoV-2 coronavirus, has produced a pandemic infecting more than 10 million people and causing more than 500,000 deaths worldwide, challenging not only healthcare systems but also the culture and economy of the population [1].

SARS-CoV-2 causes COVID-19 (coronavirus disease 19), which in most cases is an asymptomatic or mildly symptomatic condition that resolves without therapy or with minor supportive treatment. In some cases, SARS-CoV-2 can cause pneumonia, which in $20 \%$ may be moderate in severity. However, a subgroup of patients with COVID-19 pneumonia develops rapidly progressing respiratory failure that may necessitate mechanical ventilation and support in an intensive care unit (ICU) and/or result in multi-organ and systemic manifestations in terms of sepsis, septic shock, and multiple organ dysfunction syndromes [2].

Available evidence suggests that a hyper-inflammatory syndrome (HIS) that resembles secondary hemophagocytic lymphohistiocytosis (sHLH) may have a pathogenic role $[3,4]$. This is consistent with the general knowledge about coronavirus (CoV) infections, where the immune response has been shown to play an essential role in controlling and eliminating $\mathrm{CoV}$ infections, and maladjusted immune responses may result in immunopathology and impaired pulmonary gas exchange [5].

At the time of conducting the study, treatment was mainly symptomatic, with oxygen therapy representing the major treatment intervention for patients with severe infection. Several antiviral treatments have been used 
such as lopinavir/ritonavir, chloroquine, hydroxychloroquine, remdesivir, and alpha-interferon. No vaccine is currently available. Based on the observation that acute, lifethreatening respiratory injury associated with an over-exuberant cytokine release occurred in some patients infected by SARS-CoV-2, treatment strategies aiming to break down this hyper-inflammatory response were considered. Elevated levels of blood interleukin-6 (IL-6) have been identified as a one of the risk factors associated with severe COVID-19 [5].

In this context, tocilizumab, a humanized IgG1 monoclonal antibody targeting the IL-6 receptor, was postulated as a suitable treatment option [6]. Preliminary experiences with tocilizumab showed promising results with improvements seen in clinical symptoms, oxygenation status, and inflammatory laboratory parameters [7]. This limited evidence together with a strong mechanistic rationale, plus current knowledge on the role of anti-IL6 inhibitors in the treatment of cytokine release syndromes, supported the progressively extended use of tocilizumab in clinical practice. Simultaneously numerous clinical studies, including clinical trials, were initiated worldwide with the aim to assess the potential efficacy of tocilizumab in preventing the fatal consequences of acute respiratory and multiorgan failure associated with acute respiratory syndrome due to severe COVID-19.

At the time of launching our study (3 March 2020), many uncertainties existed about the actual benefits and risks of tocilizumab in the treatment of SARS-CoV-2 pneumonia. To increase knowledge on the actual role of tocilizumab in SARS-CoV-2 pneumonia, we conducted a retrospective multicentre cohort study. Our study aimed to describe our experience with a series of consecutive COVID-19 patients treated with tocilizumab in 18 tertiary hospitals in Spain and compare the outcomes of this cohort with those observed in a similar cohort of SARS-CoV-2 infected patients who did not receive tocilizumab to identify patients who will most benefit from its use.

\section{METHODS}

\section{Design, Study Period, and Subjects}

This was a retrospective, observational cohort study performed in 18 tertiary Spanish hospitals including patients with severe COVID-19.

The study population were adult patients ( $\geq 18$ years) with COVID-19, confirmed by PCR on nasopharyngeal swab, who were consecutively admitted to the participating hospitals between 3 March and 20 April 2020.

Patients were included consecutively according to the date of admission to hospital until the planned sample size was met in a competitive manner. Eighteen centres contributed data to both cohorts (Online Appendix Table 1). Eligible patients were hospitalised patients outside the intensive care units (ICU) with documented pneumonia (by either imaging and/or the presence of rales/crackles on physical examination) with severe respiratory failure. Severe respiratory failure was defined by presence of a Brescia-COVID Scale score of 2 (i.e. patients on oxygen therapy plus one of the following criteria: (1) the patient had dyspnoea or staccato speech at rest or after minimum activity; (2) respiratory rate $>22$ with $>61 / \mathrm{min}$ $\mathrm{O}_{2}$; (3) significant worsening of the chest X-ray) or an score of 3 (i.e. the patient required highflow nasal ventilation, continuous positive airway pressure (CPAP), or non-invasive ventilation (NIV) because of rapid deterioration of respiratory exchanges without immediate possibility of invasive ventilation (Fig. 1) [8]; and at least one of these parameters: IL6 $>40 \mathrm{pg} / \mathrm{ml}$, or increasing lactate dehydrogenase (LDH) or $\mathrm{LDH}>$ twice the upper limit of normal, increasing $\mathrm{C}$ reactive protein (CRP), D-dimer (DD) $>1500 \mathrm{ng} / \mathrm{ml}$, lymphocytes $<1200 / \mu \mathrm{l}$, or ferritin $>500 \mathrm{ng} / \mathrm{ml}$. We excluded patients < 18 years old and those who died within $24 \mathrm{~h}$ after admission to hospital or after developing inclusion criteria. Of these, patients who received tocilizumab therapy according to clinical practice were assigned to the tocilizumab cohort, whilst patients who did not were assigned to the control cohort. 


\begin{tabular}{|l|l|}
\hline \multicolumn{3}{|l|}{ Brescia-COVID respiratory severity scale } \\
\hline 0 & Ambient air \\
\hline 1 & Oxygen therapy \\
\hline 2 & $\begin{array}{l}\text { Oxygen therapy plus } 1 \text { of the following criteria: } \\
\text { a) The patient has dyspnoea or STACCATO SPEECH (inability to count rapidly to } 20 \text { after taking a } \\
\text { deep breath) at rest or after minimum activity (sitting down on bed, standing up, speaking, } \\
\text { swallowing, coughing) } \\
\text { b) Respiratory rate }>22 \text { with }>6 \mathrm{~L} / \text { minute } \mathrm{O} 2 \\
\text { c) PaO2 }<65 \mathrm{mmHg} \text { with }>6 \mathrm{~L} / \text { minute } \mathrm{O} 2 \\
\text { d) Significant worsening of chest x-ray (increased compactness and extension of infiltrate) }\end{array}$ \\
\hline 3 & The patient requires high-frequency nasal ventilation (HFNC), CPAP or $\mathrm{NIV}$ \\
\hline 4 & The patient is intubated for CPAP or pressure support \\
\hline 5 & The patient is under controlled mechanical ventilation; PaO2/FiO2 $>150 \mathrm{mmHg}$ \\
\hline 6 & The patient is under controlled mechanical ventilation; PaO2/FiO2 $\leq 150 \mathrm{mmHg}$ \\
\hline 7 & $\begin{array}{l}\text { The patient is under controlled mechanical ventilation; PaO2/FiO2 } \leq 150 \mathrm{mmHg} \text { and intravenous } \\
\text { infusion of neuromuscular blockers }\end{array}$ \\
\hline 8 & $\begin{array}{l}\text { The patient is under controlled mechanical ventilation; PaO2/FiO2 } \leq 150 \mathrm{mmHg} \text { and one of the } \\
\text { following: } \\
\text { a) Prone position } \\
\text { b) ECMO }\end{array}$ \\
\hline
\end{tabular}

Fig. 1 Brescia-COVID respiratory severity scale

\section{Data Collection}

Epidemiological, clinical, pharmacological, laboratory, and radiological data were extracted from medical records using a standardised data collection form. The patients were followed according to clinical practice. Data were collected from days $1,3,7,15$, and 28 post-inclusion. Adverse events possibly or probably related to tocilizumab treatment were collected for treated patients. All data were included by a primary reviewer and subsequently checked by two senior physicians.

\section{Laboratory Procedures}

Routine blood examinations included a complete blood count, coagulation profile, serum biochemical tests (including LDH), CRP, DD, IL6 , and serum ferritin. Chest radiographs or computed tomography (CT) scans were also done for all inpatients.

\section{Definition of the Outcome}

The primary outcome of the study was in-hospital mortality. The outcome of patients treated with tocilizumab was compared to that of those who did not receive tocilizumab. In patients from the non-treated cohort, baseline day (day 0 ) was defined as the first day that the patient fulfilled the inclusion criteria established in the protocol. In patients from the tocilizumab cohort, baseline day was considered the day of initiation of treatment with tocilizumab.

\section{Definition of the Exposure}

Exposure to tocilizumab was defined as the use of intravenous tocilizumab at any time during the hospital admission. The decision to prescribe tocilizumab was at the discretion of the treating physician.

Details of tocilizumab use (including the timing of initiation, dosing, and number of doses) were recorded. Likewise, the choice of COVID-19 treatments other than tocilizumab was at the discretion of the treating physician, 
although based on national and local recommendations for COVID-19 management.

For the main analysis, we generated a variable with the following mutually exclusive categories: "non-use of tocilizumab drug" (control cohort) and "use of tocilizumab drug" (treatment cohort). Subsequently, for the treatment cohort, we disaggregated the latter into two different subgroups: the 'with concomitant glucocorticoids or corticoids (steroids)' and 'no concomitant steroids' groups. Concomitant use of steroids was defined as initial use within the first $48 \mathrm{~h}$ after tocilizumab administration (steroid-48 h).

\section{Statistical Analysis}

Categorical variables are described with frequencies and percentages and continuous variables with mean (standard deviation, SD) and median [interquartile range (IQR): 25th-75th percentiles], and the survival function is described using the Kaplan-Meier function.

We used standardised differences, defined as differences between groups divided by pooled standard deviation to assess heterogeneity between both cohorts for baseline covariates. The inverse probability of the treatment weights (IPTW) approach [9] was used to create a pseudo-population in which the two groups were balanced across baseline covariates. The stabilised weights were calculated using propensity scores (PS) [10] obtained from a logistic regression model aimed to minimise the between arms standardised differences [11]. The baseline covariates included in the final model were gender, age, hypertension, neurological exploration, diabetes mellitus, WHO ordinal scale, time from symptoms, confirmed infection, lymphocytes, neutrophils, platelets, prothrombin activation, temperature, $\mathrm{LDH}$, and baseline medication use of angiotensin converting enzyme (ACEs) inhibitors, lopinavir-ritonavir, hydroxychloroquine, corticosteroids, interferon, non-steroidal anti-inflammatory drugs, moxifloxacin, remdesivir, and azithromycin. Covariate balance was assessed using the standardised differences with the goal to achieve values $<0.10$ using the IPTW to define insignificant differences in potential confounders, which was achieved by most, but not by all, baseline covariates. However, for some authors $<0.20$ might also be acceptable $[12,13]$ and only IL-6 remained unbalanced $(S T D=0.42)$ with no possible PS correction given the high number of missing data $(\approx 51 \%)$.

Since there were missing data for some key covariates needed for the PS calculations, the following variables (all with $<10 \%$ missing data) were imputed using the EM algorithm, which relies on the flexible and reasonable missing at random assumption: WHO ordinal scale, time from symptoms, lymphocytes, neutrophils, platelets, haemoglobin, CRP, DD, temperature, $\mathrm{LDH}$, and prothrombin activation.

Baseline categorical data were compared using the chi-square test and continuous variables using ANOVA with rank-transformed data for raw and IPTW adjusted analyses. Raw and IPTW-adjusted regression models were used to estimates risks [hazard ratio (HR) with 95\%CI $(95 \% \mathrm{CI})]$ for time-to-event variables.

In all statistical analyses, we applied a twosided type I error of 5\%. SPSS v.25 (IBM Corp., Armonk, NY) and SAS v9.4 (Cary, NC, USA) software was used.

\section{Ethics}

The study was approved by the Spanish Regulatory Authority (Spanish Agency of Medicines and Medical Devices, AEMPS) and by the Research Ethics Committee (REC) at Hospital Universitario Puerta de Hierro-Majadahonda (FIB-TOC-2020-01), and a waiver for informed consent was granted. The study protocol was submitted to the local RECs at each study site and in line with Spanish legislation; some provided their own additional approval while others did not need to undergo the full review and approval process, i.e. they recognised the initial REC approval. The study complied with the provisions in European Union (EU) and Spanish legislation on data protection and the Declaration of Helsinki 2013. 


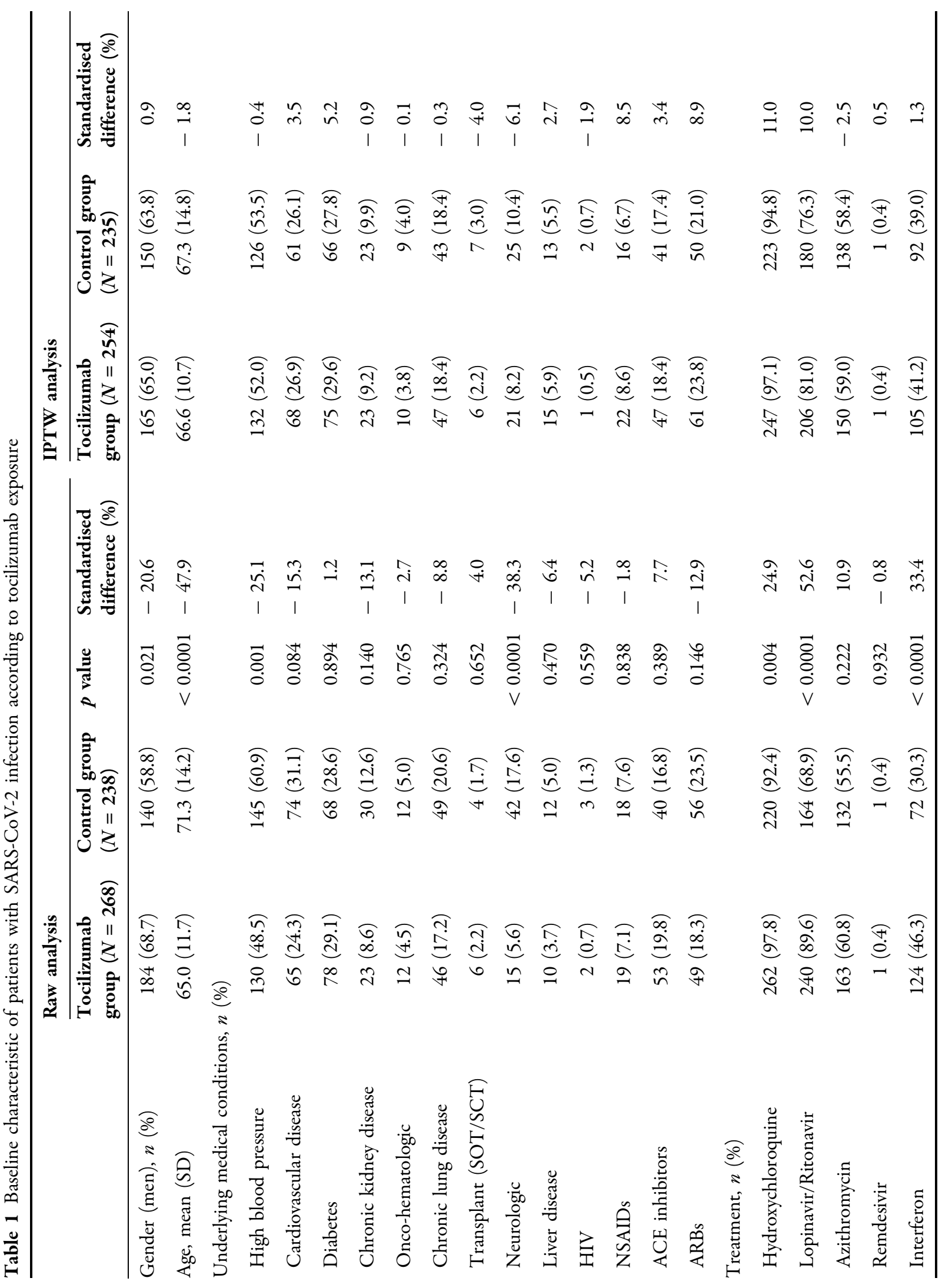




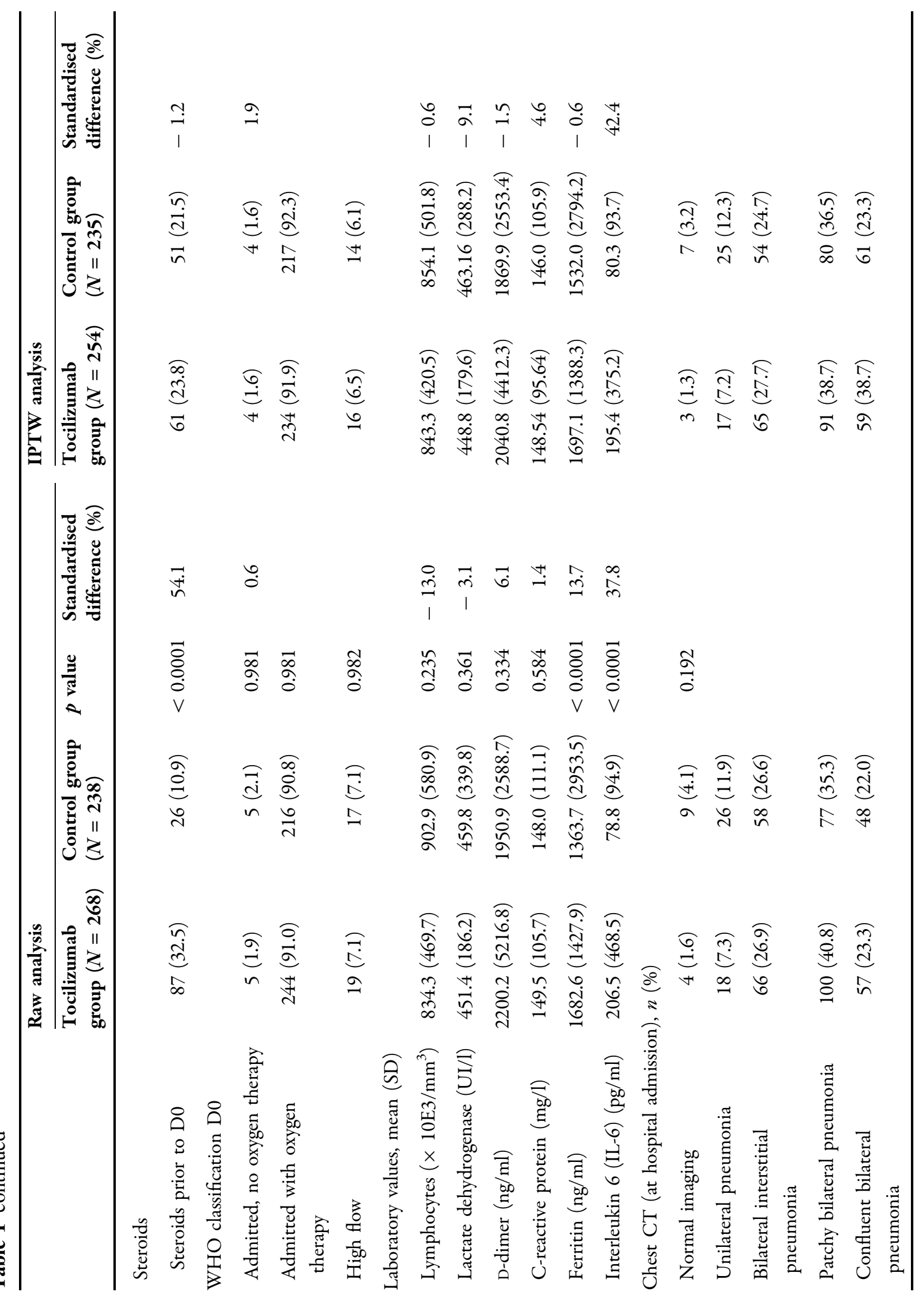




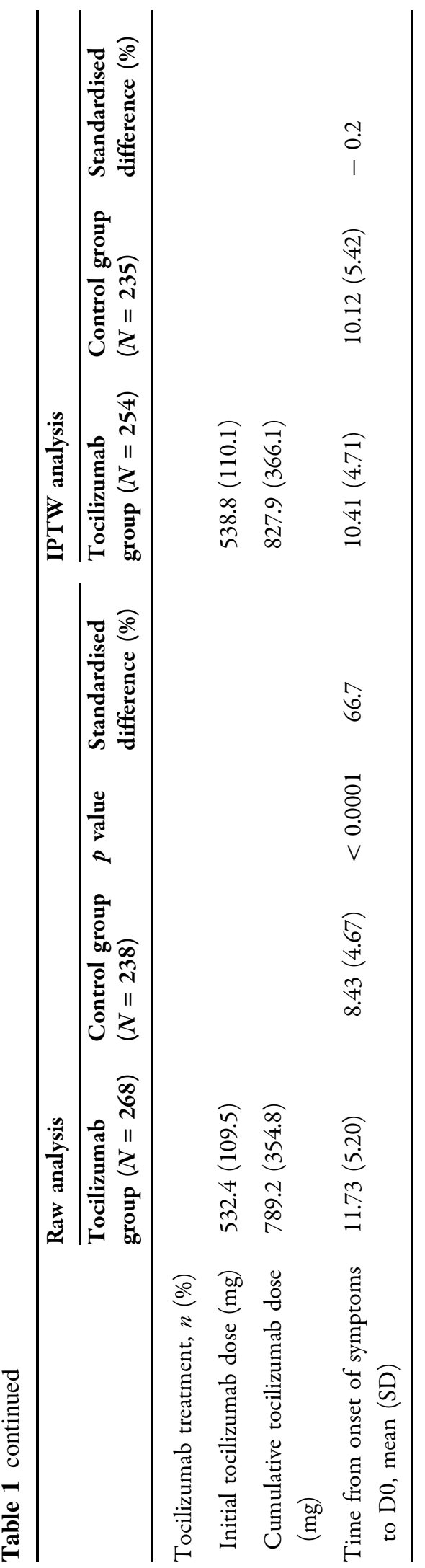

\section{RESULTS}

During the study period, 506 consecutive patients with COVID-19, respiratory insufficiency, and increased inflammatory parameters were included. Among them, 268 were treated with tocilizumab and 238 patients were not. Twenty-four patients who died in the first $24 \mathrm{~h}$ after developing inclusion criteria were excluded.

\section{Baseline Clinical Characteristics}

Characteristics of both cohorts are shown in Table 1. Median time to tocilizumab from the onset of symptoms was 11 days (IQR 8-14 days).

Among 268 patients treated with tocilizumab, 22 (8.2\%) received 3 doses, 92 (34.3\%) received 2 doses, and the remaining 154 (57.4\%) received only one dose. Median initial tocilizumab dose was $600 \mathrm{mg}$ (IQR 400-600). Median time from first dose to second dose was 1 day (IQR 1-1) and from first dose to third dose was 2 days (IQR 1-3). Median cumulative dose of tocilizumab was $600 \mathrm{mg}$ (IQR 600-1000).

A PS was developed to estimate each patient's probability of receiving tocilizumab given their baseline characteristics and reduce selection bias. Characteristics of the treatment and control cohort after adjustment by IPTW are shown in Table 1.

\section{In-Hospital Mortality of Patients Treated with Tocilizumab Compared to Patients Not Treated with Tocilizumab}

Global hospital mortality was $23.7 \%$. Characteristics of survivors and non-survivors are shown in Online Appendix Table 2.

Mortality was lower in patients treated with tocilizumab than in controls $[16.8 \%$ versus $31.5 \%$, HR $0.514 \quad$ (95\% CI $0.355-0.744)$, $p<0.001$ ] (Table 2). Tocilizumab treatment reduced mortality by $14.7 \%$ relative to no tocilizumab treatment (RRR 46.7\%). We calculated a number needed to treat of 7 . Differences in mortality persisted after applying the PS adjusted for tocilizumab treatment [weighted HR $0.741 \quad(95 \% \quad$ CI $\quad 0.619-0.887), \quad p=0.001]$ 
Table 2 Association between tocilizumab treatment and mortality in patients with SARS-COV-2 infection, according to tocilizumab and steroid- $48 \mathrm{~h}$ exposure

\begin{tabular}{|c|c|c|c|c|c|c|}
\hline \multirow[t]{2}{*}{ All } & \multicolumn{2}{|c|}{ Number of events } & \multicolumn{2}{|l|}{ Ratio } & \multicolumn{2}{|l|}{ IPTW ratio } \\
\hline & $\begin{array}{l}\text { Tocilizumab, } \\
N=268\end{array}$ & $\begin{array}{l}\text { No tocilizumab, } \\
N=238\end{array}$ & HR (95\% CI) & $p$ value & wHR (95\% CI) & $p$ value \\
\hline Deaths & $45(16.8 \%)$ & $75(31.5 \%)$ & $0.514(0.355-0.744)$ & $<0.001$ & $0.741(0.619-0.887)$ & 0.001 \\
\hline Steroid- $48 \mathrm{~h}$ & $N=119$ & $N=87$ & & & & \\
\hline Deaths & $13(10.9 \%)$ & $35(40.2 \%)$ & $0.511(0.352-0.741)$ & $<0.001$ & $0.600(0.449-0.804)$ & 0.006 \\
\hline $\begin{array}{l}\text { No steroid- } \\
\quad 48 \mathrm{~h}\end{array}$ & $N=149$ & $N=151$ & & & & \\
\hline Deaths & $32(21.5 \%)$ & $40(26.5 \%)$ & $0.713(0.447-1.137)$ & 0.345 & $0.830(0.650-1.048)$ & 0.115 \\
\hline
\end{tabular}

$H R$ hazard ratio, IPTW inverse probability of treatment weighting, $w H R$ weighted hazard ratio, steroid- $48 \mathrm{~h}$ treated with steroids in the $48 \mathrm{~h}$ following inclusion

*Weighted hazard ratios and 95\% confidence intervals were obtained by inverse probability treatment weighting

(Table 2). Figure 2 demonstrates differences in the probability for survival for patients with SARS-CoV2 infection, according to tocilizumab treatment $(\log$-rank $p<0.001)$.

\section{Tocilizumab Effect in Subgroups According to Baseline Characteristics}

The effect of tocilizumab treatment on mortality in different subsets of patients was consistent with a protective effect. Figure 3 shows different effects of tocilizumab according to different values of variables that displayed significant interaction. Patients $>65$ years old with lymphocyte counts $<1000$ cells/ $\mu$ l, hypertension, and cardiovascular disease seem to benefit the most from tocilizumab treatment.

\section{Effect of Tocilizumab in Patients with Post-Baseline Steroid Treatment}

One-hundred nineteen (44.4\%) patients in the tocilizumab arm and $87(36.6 \%)$ in the control arm were treated with steroids in the $48 \mathrm{~h}$ following inclusion (steroid-48). Among them $71.3 \%$ patients received steroids in pulses, and $28.7 \%$ non-pulsed steroids. Characteristics of patients according to tocilizumab and steroid$48 \mathrm{~h}$ exposure are shown in Online Appendix Table 3.
An interaction was found between tocilizumab and steroid-48 $\mathrm{h}$ use (interaction $p=0.094)$. Among patients treated with steroid$48 \mathrm{~h}$, mortality was lower in those treated with tocilizumab than in those treated with steroid$48 \mathrm{~h}$ alone [10.9\% vs. 40.2\%, HR 0.511 (95\% CI $0.352-0.741), p=0.036$; weighted HR $0.6(95 \%$ CI 0.449-0.804), $p<0.001$ ] (Table 2).

Tocilizumab with steroid-48 $\mathrm{h}$ treatment reduced mortality by $29.1 \%$ relative to no tocilizumab treatment (RRR 72.8\%). We calculated a number needed to treat of 4 . Figure 4 shows differences in probability of survival according to steroid exposure in patients treated with tocilizumab.

\section{Adverse Events}

Adverse events were monitored during the study period in the tocilizumab group. Although up to $32.6 \%$ in the tocilizumab group compared to $30.3 \%$ in the control group had an increase in SGOT/AST above the upper limit of normal, there were no significant differences among the study groups. A total of $11(4.1 \%)$ patients in the tocilizumab group had serious adverse reactions, including hepatotoxicity with increased liver enzymes (3 patients) or bilirubin ( 2 patients), thrombocytopenia (1 patient), catheter-related superficial thrombophlebitis (2 patients), 


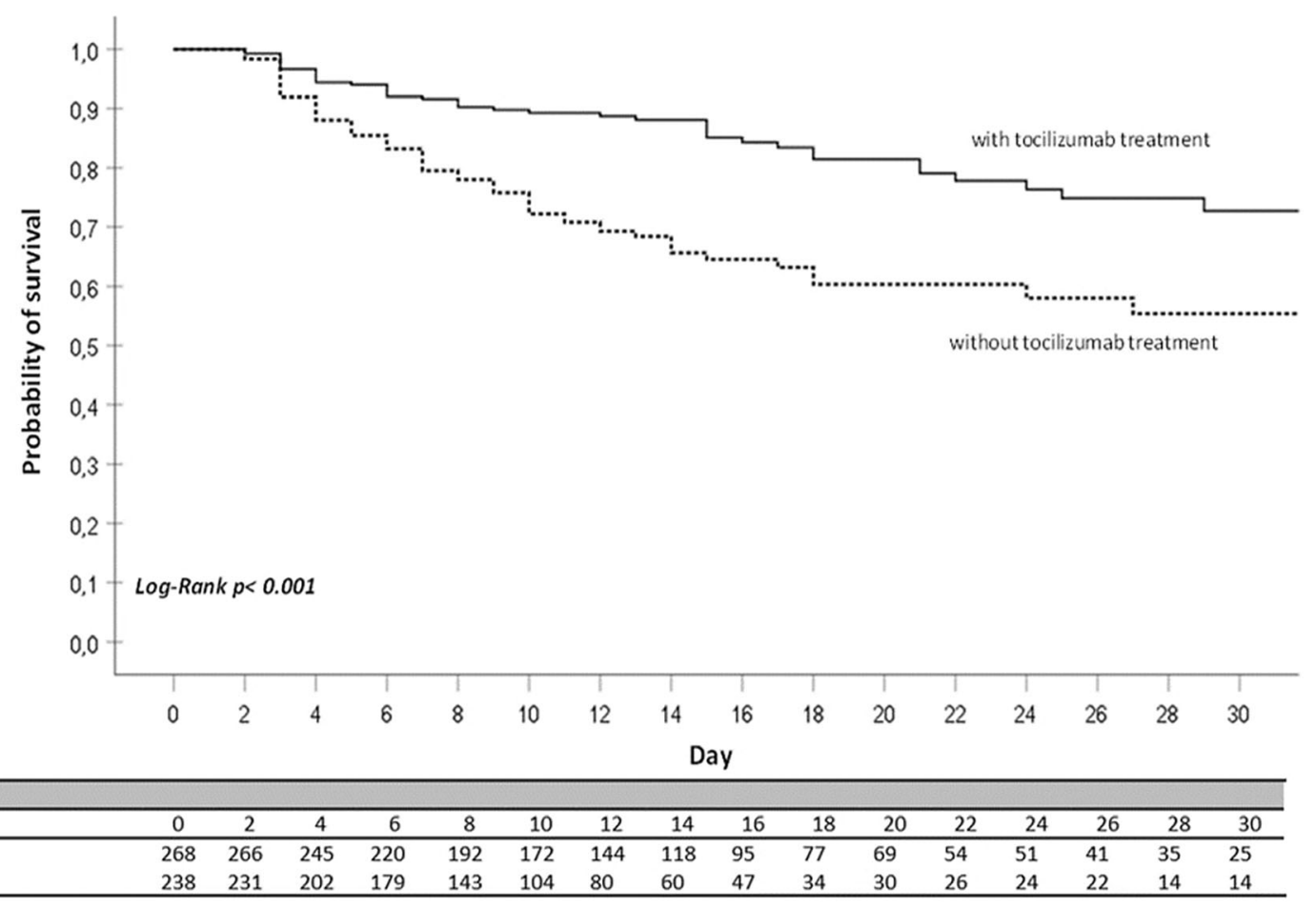

Fig. 2 Probability of survival of patients with SARS-COV-2 infection according to tocilizumab exposure. Descriptive raw analysis

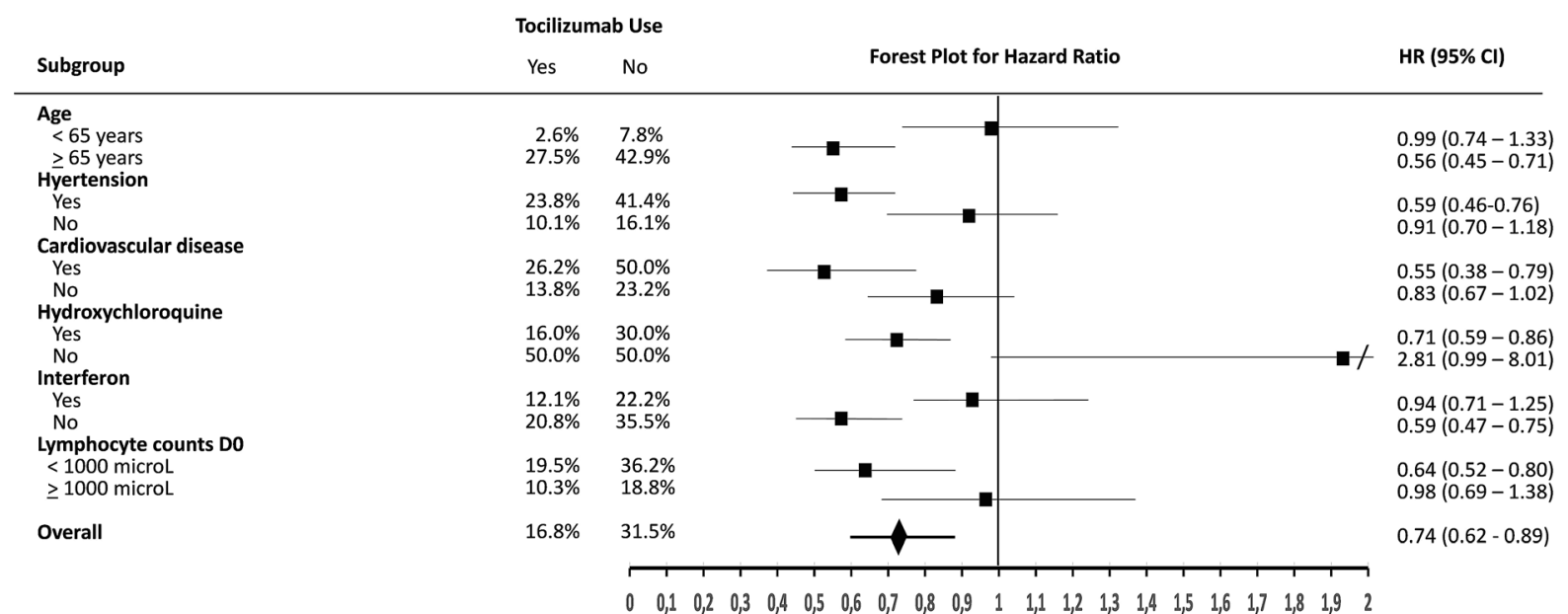

Fig. 3 Forest plot of stratified analyses for mortality showing the weighted hazard ratio from IPTW analysis of tocilizumab treatment

diarrhoea (1 patient), and headache and ocular phosphenes (1 patient). Bacteraemia without a source after tocilizumab administration was documented in one patient (0.4\%). Median follow-up time was 12 days (7-18 days).

\section{DISCUSSION}

Our results show that survival of patients with severe COVID-19 is significantly higher in patients treated with tocilizumab than in those 


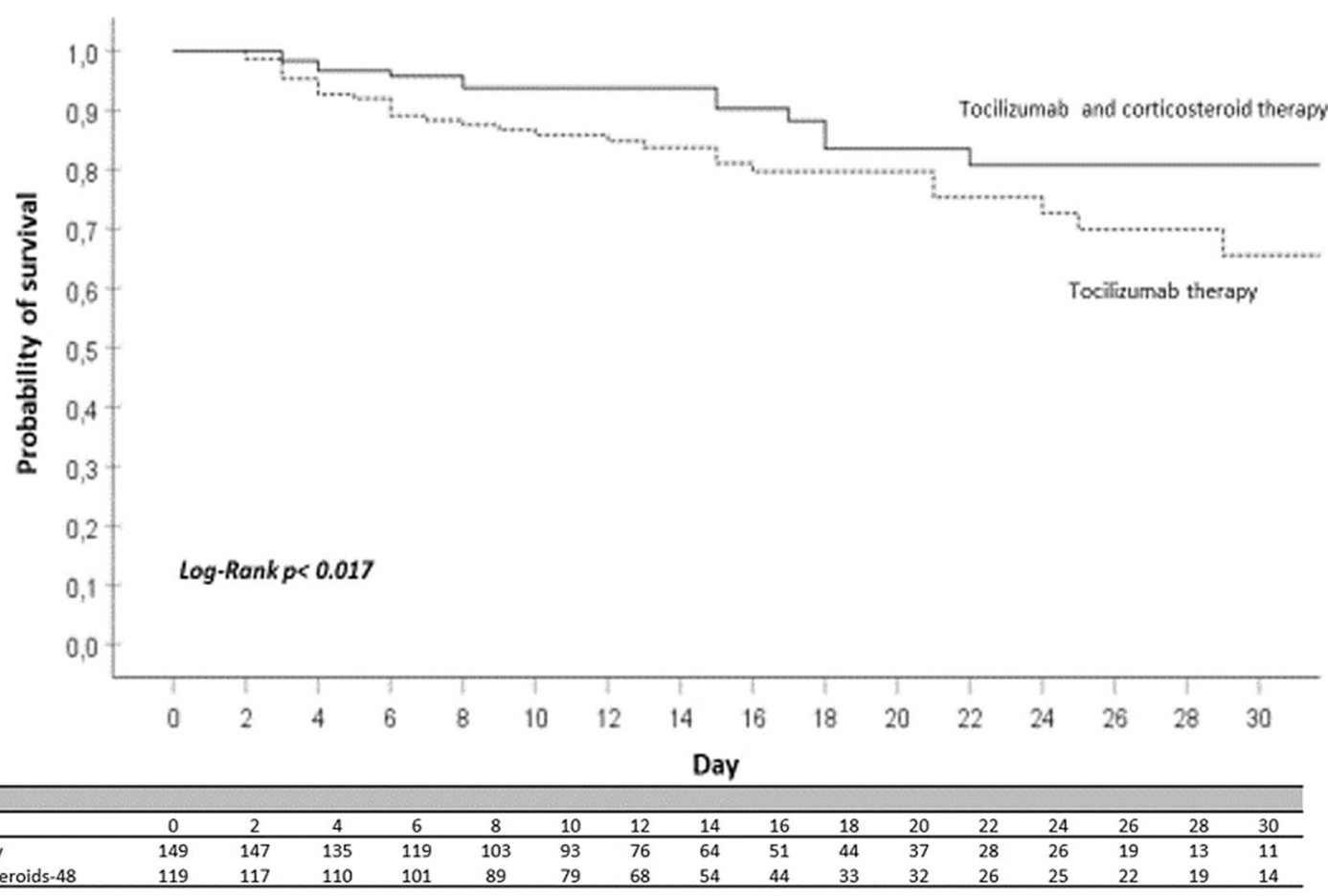

Fig. 4 Probability of survival of patients with SARS-COV-2 infection treated with tocilizumab according to steroid-48 $\mathrm{h}$ exposure. Descriptive raw analysis

not treated. In particular, mortality is reduced when tocilizumab is used concomitantly or shortly followed (first $48 \mathrm{~h}$ ) by steroids. These results come from a large cohort of 506 consecutive patients with COVID-19 pneumonia, respiratory insufficiency, and increased inflammatory parameters recruited across 18 hospitals from different regions in Spain. Of these, 268 were treated with tocilizumab whilst 238 patients were not. These results are considered clinically relevant as they contribute to the growing body of evidence supporting the use of tocilizumab in SARS-CoV-2 infection.

Our data are consistent with previously published series that report a reduction in the risk of death in patients with severe COVID-19 treated with tocilizumab compared to standard of care. Most of these are observational studies, some of them comparing tocilizumab use with a control group $[14,15,16]$.

At the time of planning our study, results from randomised controlled trials had not yet been published. Recently, the results of the first randomised, open label, controlled trial of tocilizumab versus standard of care have been published [17]. The study was prematurely interrupted because of futility (and recruitment difficulties) with one third of the initially planned study population. As recognised by the authors, this small open-label clinical trial still leaves important questions unanswered on the potential role of tocilizumab in the treatment of COVID-19.

Recently, steroid treatment has shown promise as a life-saving therapy in patients with COVID-19 [18, 19], and, indeed, dexamethasone has been granted EU regulatory approval for the treatment of COVID-19 patients on oxygen or mechanical ventilation, becoming the standard of care in clinical practice. Therefore, when analysing the effect of new treatments, it is important to consider the combined use of steroid therapy.

Prior studies do not report specifically on the combined effect of tocilizumab and post-baseline steroids, although in some of them steroids have been used concomitantly with tocilizumab in a considerable proportion of cases $[17 \%$ 
standard of care (SoC) and 30\% tocilizumab] [14], close to $100 \%$ in some cases $[20,21]$. Contrarily, in the only randomised clinical trial concomitant use was not allowed unless patients were on chronic treatment. A singlecentre Italian study jointly analysed data regarding the administration of both tocilizumab and steroids, together or separately, and suggested the need for evaluating combined use of these therapies [22]. More recently, Narain et al. published the results of a retrospective cohort study and concluded that the combination of corticosteroids with tocilizumab showed superior survival outcome compared with SoC treatment and treatment with corticosteroids alone or in combination with anakinra [23]. These results are consistent with our findings.

The timing of steroid administration in relation to tocilizumab treatment might be decisive. A small non-controlled Spanish cohort study suggests that patients treated with prior or concomitant steroid therapy had better survival results than those with steroids added at a later stage [20]. Another short observational singlecentre study shows an improvement in mortality in patients treated with tocilizumab when salvage steroids are added in a median of 2.3 days [24]. In our study, tocilizumab adds to steroid administered to non-intubated cases with severe COVID-19 during the first $48 \mathrm{~h}$ of presenting with respiratory failure despite oxygen therapy (Brescia-COVID respiratory severity scale 2 or worse).

The severity of the study population that could most benefit from treatment remains to be determined. Some authors suggest that the administration of tocilizumab is more effective in severe cases $(\mathrm{PaO} 2 / \mathrm{FiO} 2<150)$ [14], while others report better results in cases with less oxygen requirement [21].

In our study, tocilizumab showed an added benefit to that of steroids in reducing mortality of severe COVID-19. The major strength of this study was its multicentric nature, with a large number of patients included from different regions in Spain. The study also included a concurrent comparison with a non-treated cohort. The IPTW to adjust for indication bias adds to the strength of the results.
However, this is an observational study and, as such, has the inherent limitations of this kind of study. Indication bias for steroid treatment was not adjusted for. It is possible that steroid treatment was not evenly administered across the study population. This could possibly explain the high mortality in the subset of patients who received steroid- $48 \mathrm{~h}$, but not tocilizumab.

The short follow-up time prevented us from detecting important adverse events such as reactivation of latent infections in patients treated with tocilizumab, particularly in those treated with tocilizumab and steroids. Thus, safety concerns might have been underestimated. To overcome this problem, ongoing registries have been put in place to identify medium-term complications.

Although our cohort was limited to non-ICU patients, tocilizumab's effect on critical patients who already require mechanical ventilation or ICU admission deserves further investigation. One recent article by Somers et al. [25] suggests that tocilizumab in ICU patients is also beneficial, although the risk of infection is high.

\section{CONCLUSION}

Our study results show that survival of patients with severe COVID-19 is higher in patients that received tocilizumab as part of the standard of care than in those who did not. The benefits appear higher when tocilizumab is given concomitantly with steroids, mostly when given within the first $48 \mathrm{~h}$ of presenting with respiratory failure despite oxygen therapy. Nevertheless, relevant questions remain unanswered. Large, randomised, controlled studies are needed to convincingly establish the efficacy and safety of tocilizumab in the treatment of COVID-19.

\section{ACKNOWLEDGEMENTS}

We thank the members of the TOCICOV study group for their contribution to the work. 
TOCICOV Study Group Collaborators: HOSPITAL DEL MAR-PARC DE SALUT MAR. X Fernández Sala, P. Díaz Pellicer, S. Grau Cerrato. HOSPITAL UNIVERSITARIO DE PUERTO REAL. José Manuel Dodero Anillo, Alberto Romero Palacios. HOSPITAL UNIVERSITARIO VIRGEN DEL ROCÍO. Elisa Cubiles, $\mathrm{M}^{\mathrm{a}}$ Ángeles Lobo Acosta, Reyes Fresneda Gutierrez, Alicia Marían Candón, Sonsoles Salto Alejandre, Jose Miguel Cisneros Herreros, Elisa Cordero Matía, Carmen Infante Dominguez, Juan Carlos Crespo Rivas, Macarena López Verdugo. HOSPITAL UNIVERSITARIO DE CANARIAS. Isabel Suarez Toste. Paloma Díaz Pérez. HOSPITAL UNIVERSITARI DE BELLVITGE. Francesca Mitjavila Villero, Guillermo Suárez Cuartin, Carlota Gudiol Gonzalez, Adriana Iriarte Fuster, Mercè Gasa Galmes, Sandra Pérez, Dolores Rodríguez Cumplido. HOSPITAL UNIVERSITARIO Nra Sra. CANDELARIA. Arístides de León Gil, Emilio J. Sanz. HOSPITAL GENERAL UNIVERSITARIO DE VALENCIA. Francisco Sanz-Herrero, Francesc Puchades, Pilar Ortega-García. C.H. UNIV. DE SANTIAGO DE COMPOSTELA. José Antonio Díaz-Peromingo, Carlos Rodríguez-Moreno. HOSPITAL UNIVERSITARIO CLÍNICO SAN CARLOS Daniel Lozano, Ana Terleira. HOSPITAL UNIVERSITARIO TORRECÁRDENAS. María Estela González Castro, Sergio Ferra Murcia, Elena María Gázquez Aguilera. HOSPITAL UNIVERSITARIO DE LA PRINCESA. Elena PintosSanchez, Pablo Zubiaur, Elena Santos-Molina, Marcos Navares-Gómez, Gina Mejia Abril. HOSPITAL CENTRAL DE LA DEFENSA GÓMEZ ULLA. Amelia García-Luque, Miguel Puerro-Vicente, María Jesús Sánchez Carrillo. HOSPITAL UNIVERSITARIO RAMÓN Y CAJAL. M Ángeles Gálvez Múgica, Mónica Aguilar Jiménez, Cristina Sánchez Díaz. HOSPITAL UNIVERSITARIO PUERTA DE HIERRO. Cristina Avendaño Solá, Patricia Mills-Sánchez, Manuel Valle, Gustavo Centeno, Concepción Payares, Antonio F. Caballero Bermejo, Elena Diago, Rosa Malo de Molina, Juan Antonio Vargas Nuñez, Elena Muñez, Antonio Ramos. HOSPITAL UNIV. GERMANS TRIAS I PUJOL Ana María Barriocanal, Ana Pilar Pérez-Acevedoc, Melani NúñezMontero. HOSPITAL UNIVERSITARIO GREGORIO MARAÑON. María Olmedo, Sofia De la Villa. HOSPITAL UNIVERSITARIO VIRGEN DE
LA VICTORIA Enrique Nuño Álvarez, MI Lucena. HOSPITAL COSTA DEL SOL. Josefa Andrea Aguilar García, José Javier García Alegría.

Funding. No funding or sponsorship was received for this study or publication of this article. The Rapid Service Fee was funded by the authors.

Authorship. All named authors meet the International Committee of Medical Journal Editors (ICMJE) criteria for authorship for this article, take responsibility for the integrity of the work as a whole, and have given their approval for this version to be published.

Authorship Contribution. Conceptualization and study design: ASL, BRA, AFC. Methodology: BRA, AFC, ASL, F-T. Data collection: all authors. Data interpretation: ASL, BRA, AFC, FT. Writing first draft: ASL, BRA, AFC. Critical revision for important intellectual content: all authors. Final approval: All authors. All authors agree to be accountable for all aspects of the work by ensuring that questions related to the accuracy or integrity of any part of the work will be appropriately investigated and resolved. AFC and BRA had full access to all the data in this study and take complete responsibility for the integrity of the data and the accuracy of the data analysis.

Prior Presentation. This manuscript is based on work that has been previously presented at medRxiv, posted 10 September 2020 (https:// www.medrxiv.org/content/10.1101/2020.09. 07.20189357v2).

Disclosures. Belén Ruiz-Antorán, Aránzazu Sancho-López, Ferrán Torres, Víctor MorenoTorres, Itziar de Pablo-López, Paulina GarcíaLópez, Francisco Abad-Santos, Clara M. RossoFernández, Ana Aldea-Perona, Eva Montané, Ruth M. Aparicio-Hernández, Roser Llop-Rius, Consuelo Pedrós, Paloma Gijón, Carolina Hernández-Carballo, María J Pedrosa-Martínez, Consuelo Rodríguez-Jiménez, Guillermo PradaRamallal, Lourdes Cabrera-García, Josefa A. Aguilar-García, Rocío Sanjuan-Jimenez, Evelyn 
I. Ortiz-Barraza, Enrique Sánchez-Chica and Ana Fernández-Cruz declare no conflicts of interest.

Compliance with Ethics Guidelines. The study was approved by the Spanish Regulatory Authority (Spanish Agency of Medicines and Medical Devices, AEMPS) and by the Research Ethics Committee (REC) at Hospital Universitario Puerta de Hierro-Majadahonda (FIB-TOC2020-01), and a waiver for the informed consent was granted. The study protocol was submitted to the local RECs at each study site and in line with Spanish legislation, some provided their own additional approval while others did not need to undergo the full review and approval process, i.e. they recognised the initial REC approval. The study complied with the provisions in European Union (EU) and Spanish legislation on data protection and the Declaration of Helsinki 2013.

Data Availability. The datasets generated during and/or analysed during the current study are available from the corresponding author on reasonable request.

Open Access. This article is licensed under a Creative Commons Attribution-NonCommercial 4.0 International License, which permits any non-commercial use, sharing, adaptation, distribution and reproduction in any medium or format, as long as you give appropriate credit to the original author(s) and the source, provide a link to the Creative Commons licence, and indicate if changes were made. The images or other third party material in this article are included in the article's Creative Commons licence, unless indicated otherwise in a credit line to the material. If material is not included in the article's Creative Commons licence and your intended use is not permitted by statutory regulation or exceeds the permitted use, you will need to obtain permission directly from the copyright holder. To view a copy of this licence, visit http:// creativecommons.org/licenses/by-nc/4.0/.

\section{REFERENCES}

1. WHO. Coronavirus disease (COVID-19) Situation Report-164 [Internet]. 2020. https://www.who. int/docs/default-source/coronaviruse/situation-rep orts/20200702-covid-19-sitrep-164.pdf?sfvrsn=ac07 4f58_2. Accessed 4 Jul 2020.

2. Cascella M, Rajnik M, Cuomo A, Dulebohn SC, Di Napoli R. Features, evaluation and treatment coronavirus (COVID-19). In: StatPearls. StatPearls Publishing; 2020.

3. Mehta P, McAuley DF, Brown M, Sanchez E, Tattersall RS, Manson JJ. COVID-19: consider cytokine storm syndromes and immunosuppression. Lancet (London, England). 2020;395:1033-4.

4. McGonagle D, Sharif K, O'Regan A, Bridgewood C. The role of cytokines including interleukin- 6 in COVID-19 induced pneumonia and macrophage activation syndrome-like disease. Autoimmun Rev. 2020;19(6):102537.

5. Li G, Fan Y, Lai Y, Han T, Li Z, Zhou P, et al. Coronavirus infections and immune responses. J Med Virol. 2020;92(4):424-32.

6. Fu B, Xu X, Wei H. Why tocilizumab could be an effective treatment for severe COVID-19? J Transl Med. 2020;18:164.

7. Xu X, Han M, Li T, Sun W, Wang D, Fu B, et al. Effective treatment of severe COVID-19 patients with tocilizumab. Proc Natl Acad Sci USA. 2020;117(20):10970-5.

8. Foca E. Linee guida sulla gestione terapeutica e di supporto per pazienti con infezione da coronavirus COVID-19. 2020; Marzo. http://www.fvcalabria. unicz.it/COVID-19/LINEE-GUIDA/linee-guida-SIMIT -marzo-2020.pdf. Accessed 1 Oct 2020.

9. D'Agostino RBJ. Propensity score methods for bias reduction in the comparison of a treatment to a non-randomized control group. Stat Med. 1998;17(19):2265-81.

10. Rosenbaum PR, RDTcro. The central role of the propensity score in observational studies for causal effects. Biometrika. 1983;70:41-55.

11. Austin PC. An introduction to propensity score methods for reducing the effects of confounding in observational studies. Multivariate Behav Res. 2011;46(3):399-424.

12. Cohen DJ. Statistical power analysis for the behavioral sciences. New York: Routledge; 2013. https:// doi.org/10.4324/9780203771587. 
13. Austin PC. Balance diagnostics for comparing the distribution of baseline covariates between treatment groups in propensity-score matched samples. Stat Med. 2009;28(25):3083-107.

14. Guaraldi G, Meschiari M, Cozzi-Lepri A, Milic J, Tonelli R, Menozzi M, et al. Tocilizumab in patients with severe COVID-19: a retrospective cohort study. Lancet Rheumatol. 2020;2(8):e474-84.

15. Martínez-Sanz J, Muriel A, Ron R, Herrera S, PérezMolina JA, Moreno S, et al. Effects of tocilizumab on mortality in hospitalized patients with COVID-19: a multicentre cohort study. Clin Microbiol Infect. 2020. https://doi.org/10.1016/j.cmi.2020.09.021.

16. Rodríguez-Baño J, Pachón J, Carratalá J, Ryan P, Jarrín I, Yllescas M, et al. Treatment with tocilizumab or corticosteroids for COVID-19 patients with hyperinflammatory state: a multicentre cohort study (SAM-COVID-19). Clin Microbiol Infect. 2020. https://doi.org/10.1016/j.cmi.2020.08.010.

17. Salvarani C, Dolci G, Massari M, Merlo DF, Cavuto S, Savoldi L, et al. Effect of tocilizumab vs standard care on clinical worsening in patients hospitalized with COVID-19 pneumonia: a randomized clinical trial. JAMA Intern Med. 2020. https://doi.org/10. 1001/jamainternmed.2020.6615.

18. Fadel R, Morrison AR, Vahia A, Smith ZR, Chaudhry $Z$, Bhargava $\mathrm{P}$, et al. Early short course corticosteroids in hospitalized patients with COVID-19. Clin Infect Dis. 2020;71(16):2114-20. https://doi. org/10.1093/cid/ciaa601.

19. Fernández-Cruz A, Ruiz-Antorán B, Muñoz-Gómez A, Sancho-López A, Mills-Sánchez P, Centeno-Soto GA, et al. A retrospective controlled cohort study of the impact of glucocorticoid treatment in SARSCoV-2 infection mortality. Antimicrob Agents
Chemother. 2020;64(9):e01168. https://doi.org/10. 1128/AAC.01168-20.

20. Campins L, Boixeda R, Perez-Cordon L, Aranega R, Lopera C, Force L. Early tocilizumab treatment could improve survival among COVID-19 patients. Clin Exp Rheumatol. 2020;38:578.

21. Gorgolas M, Cabello A, Prieto Perez L, Villar Alvarez F, Alvarez Alvarez B, Rodriguez Nieto MJ, et al. Compassionate use of tocilizumab in severe SARSCoV2 pneumonia. When late administration is too late. medRxiv [Internet]. 2020;2020.06.13. 20130088. http://medrxiv.org/content/early/2020/ 06/16/2020.06.13.20130088.abstract. Accessed 5 Oct 2020 .

22. Mikulska M, Nicolini LA, Signori A, Di Biagio A, Sepulcri C, Russo C, et al. Tocilizumab and steroid treatment in patients with COVID-19 pneumonia. PLoS ONE. 2020;15(8):e0237831.

23. Narain S, Stefanov DG, Chau AS,Weber AG, Marder G, Kaplan B, et al. Comparative survival analysis of immunomodulatory therapy for coronavirus disease 2019 cytokine storm. Chest. 2020. https://doi. org/10.1016/j.chest.2020.09.275.

24. Sanz Herrero F, Puchades Gimeno F, Ortega García P, Ferrer Gómez C, Ocete Mochón MD, García Deltoro M. Methylprednisolone added to tocilizumab reduces mortality in SARS-CoV-2 pneumonia: an observational study. J Internal Med. 2020. https://doi.org/10.1111/joim.13145.

25. Somers EC, Eschenauer GA, Troost JP, Golob JL, Gandhi TN, Wang L, et al. Tocilizumab for treatment of mechanically ventilated patients with COVID-19. MedRxiv. 2020. https://doi.org/10. 1101/2020.05.29.20117358. Update in: Clin Infect Dis. 2020. 